\title{
Stimulation of ethylene production in detached rice leaves by vanadate
}

\author{
Ching Huei Kao \\ Department of Agronomy, National Taiwan University, Taipei, Taiwan, Republic of China
}

Received 23 February 1995; accepted 29 May 1995

Key words: ethylene production, Oryza sativa, vanadate

\begin{abstract}
The effect of vanadate on ethylene biosynthesis in detached rice leaves was investigated. Vanadate at $\mathrm{pH}$ 5.0-7.0 effectively enhanced ethylene production within $3 \mathrm{~h}$ of its application. It promoted the conversion of ACC to ethylene. Treatment with vanadate did not decrease ACC level until late stage of incubation, i.e. at $12 \mathrm{~h}$ after incubation. Molybdate, an inhibitor of phosphatase had no or much less stimulatory effect on ethylene production than did vanadate at comparable concentrations. Azide, an inhibitor of $F_{1}$-ATPase, inhibited ethylene production in detached rice leaves. FC and vanadate were observed to be synergisticly increased ethylene production in detached rice leaves. In conclusion, plasma membrane $\mathrm{H}^{+}$-ATPase does not seem to be involved in ethylene biosynthesis in detached rice leaves.
\end{abstract}

Abbreviations: $\mathrm{ACC}=1$-Aminocyclopropane-1-carboxylic acid; $\mathrm{FC}=$ Fusicoccin

\section{Introduction}

Zoglauer et al. [15] proposed that changes in the activity of plasma membrane $\mathrm{H}^{+}$-ATPase may be involved in the induction of biosynthesis of ethylene. An effect of transmembrane proton gradients in the conversion of ACC to ethylene has been observed in vivo and in isolated vacuoles [7, 11]. In our previous work, we have shown that FC, which is known to induce the secretion of proton via plasma membrane $\mathrm{H}^{+}$-ATPase [10], stimulated ethylene production in detached rice leaves [3]. Vanadate is recognized to act as an inhibitor of the plasma membrane $\mathrm{H}^{+}$-ATPase [13] and has been shown to inhibit ethylene production in tomato hypocotyls [9]. In order to examine the possibility that changes in the activity of the plasma membrane $\mathrm{H}^{+}$-ATPase may be involved in the induction of ethylene synthesis, we characterized the effects of vanadate on the biosynthesis of ethylene in detached rice leaves.

\section{Materials and methods}

Seedlings of rice (Oryza sativa cv. Taichung Native 1) were grown in hydroponic culture as described previously [4]. The apical $3 \mathrm{~cm}$ of the third leaves of 12day-old seedlings were used for the experiments. Ten segments of rice leaves, weighing about $45 \mathrm{mg}$, were floated on $10 \mathrm{~mL}$ of test solution in a Petri dish. All samples were kept at $27^{\circ} \mathrm{C}$ in the dark. The standard sodium vanadate solution contained $20 \mathrm{~m} M$ potassium phosphate adjusted by $\mathrm{KOH}(1 N)$ to $\mathrm{pH} 5.0$.

After various periods of incubation, the leaf samples were transferred to a $14-\mathrm{mL}$ test tube. The tubes were sealed with serum caps. After $1 \mathrm{~h}$ of incubation at $27{ }^{\circ} \mathrm{C}$ in the dark, a $1 \mathrm{~mL}$ gas sample was withdrawn from the headspace of each test tube. Ethylene was assayed with a gas chromatograph equipped with an alumina column and a flame ionization detector. Absolute levels of ethylene varied among experiments because of seasonal effects. However, the patterns of responses to vanadate were reproducible.

ACC was extracted and quantitated as described previously [8]. 
All experiments were repeated at least three times. Similar results and identical trends were obtained on each occasion. The data obtained here are all from a single experiment.

\section{Results}

The effect of the concentration of vanadate on ethylene production in detached rice leaves in darkness is presented in Fig. 1. Increasing concentration of vanadate from 0.1 to $1 \mathrm{~m} M$ progressively enhanced the production of ethylene. Vanadate-promoted ethylene production by detached rice leaves was affected by $\mathrm{pH}$ (Fig. 2). Vanadate buffered to $\mathrm{pH}$ values ranging from 5 to 7 was effective in enhancing ethylene production. However, vanadate at $\mathrm{pH} 4.0$ and 8.0 had no and less effect, respectively, on ethylene production.

Figure 3 shows the changes with time in rates of the production of ethylene in detached rice leaves treated with $1 \mathrm{~m} M$ vanadate. Promotion of the production of ethylene by vanadate was detected at $3 \mathrm{~h}$ after the start of incubation in the dark. Increased duration of treatment with vanadate increased the production of ethylene by detached rice leaves. Incubation periods longer than $6 \mathrm{~h}$ did not result in higher rate of ethylene production.

The rate of production of ethylene is generally controlled by the level of ACC, the immediate precursor of ethylene [14]. In control leaves levels of ACC increased with increasing time of incubation (Fig. 3). Treatment with vanadate had no effect on ACC levels within $3 \mathrm{~h}$ and $9 \mathrm{~h}$, but resulted in lower levels of ACC than the controls at $12 \mathrm{~h}$ after incubation (Fig. 3). These results suggest that an enhanced rate of production of ethylene in vanadate-treated segments of rice leaves is unlikely regulated by the promotion of the synthesis of $\mathrm{ACC}$. To test if the conversion of ACC to ethylene is promoted by vanadate, we measured the production of ethylene in the presence of a saturating concentration of ACC $(10 \mathrm{mM})$. As indicated in Fig. 4 , the conversion of ACC to ethylene was promoted by vanadate during a 12-h incubation.

Molybdate, an inhibitor of acid phosphatase (which may also be inhibited by vanadate) but not of $\mathrm{H}^{+}$. ATPase $[5,6,12]$, had no or much less stimulatory effect than did vanadate at comparable concentrations (Fig. 5). Azide is among several potent inhibitors of mitochondrial type $F_{1}$-ATPase [1]. Contrary to the effect of vanadate, azide inhibited ethylene production by detached rice leaves (Fig. 5). All these results

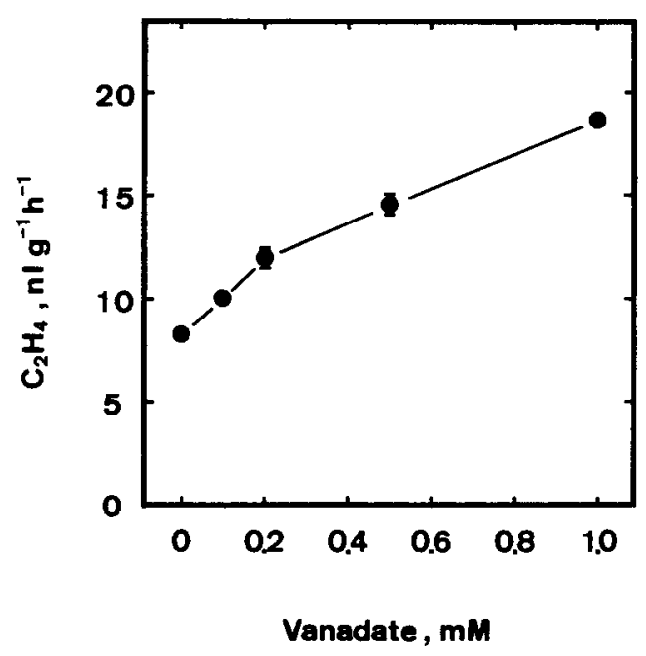

Fig. 1. Effect of vanadate concentration on ethylene production in detached rice leaves. Ethylene production was assayed after $6 \mathrm{~h}$ incubation in darkness. Bars represented standard errors $(n=4)$.



Fig. 2. Effect of $\mathrm{pH}$ on vanadate-induced ethylene production in detached rice leaves. Leaf segments were incubated in either water or $1 \mathrm{~m} M$ vanadate contained $20 \mathrm{~m} M$ potassium phosphate adjusted to various $\mathrm{pH}$. Ethylene production was assayed after $6 \mathrm{~h}$ incubation in darkness. Bars represented standard errors $(n=4)$.

indicate that the vanadate effect on ethylene production was not a consequence of nonspecific phosphohydrolase inhibition.

Figure 6 shows the effect of fusicoccin (FC) on vanadate-enhanced ethylene production in detached rice leaves. A synergistic effect on the increase of ethylene production was observed when FC and vanadate were applied together. 


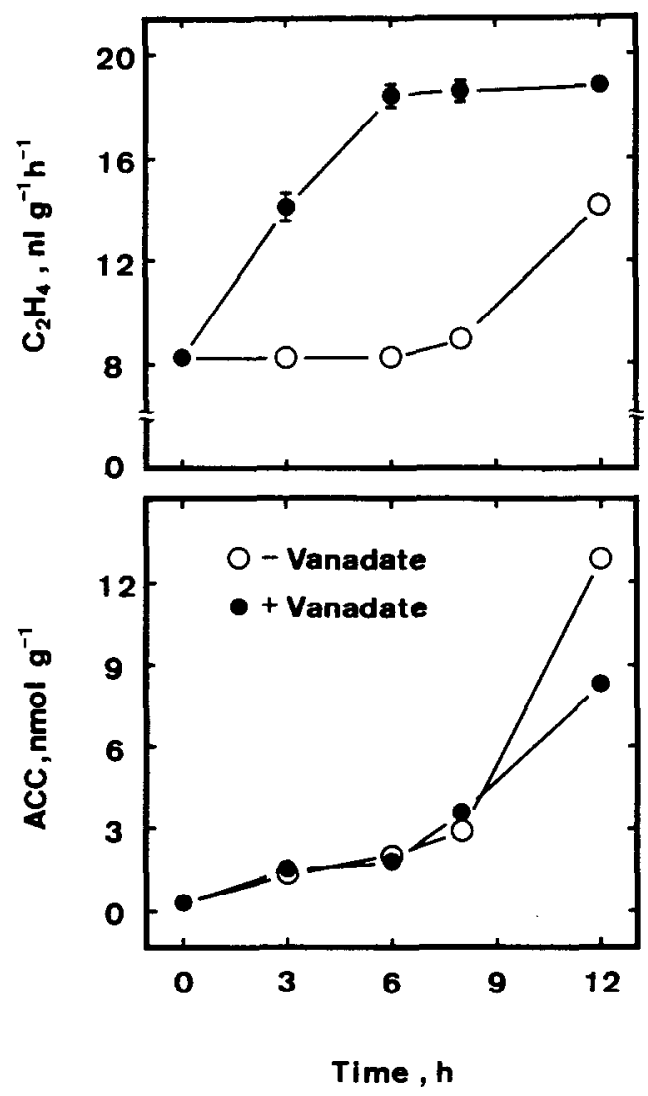

Fig. 3. Changes with time in rates of ethylene production (upper) and levels of ACC (lower) in segments of rice leaves treated with vanadate. Segments were treated with either water or $1 \mathrm{mM}$ vanadate in darkness. Production of ethylene and levels of ACC were assayed at the times indicated $(n=4)$.

\section{Discussion}

Vanadate is known to be a potent inhibitor of the plasma membrane $\mathrm{H}^{+}$-ATPase [13] and has been reported to inhibit the production of ethylene in tomato hypocotyls [9]. Unexpectedly we found that vanadate stimulated the production of ethylene in detached rice leaves. Since the increase of ethylene production in rice leaves is rapid following excision, one may argue that the effect of vanadate on ethylene production is probably a wound or other stress response. When detached leaves were used to determine ethylene production, wounding is always a problem. However, in the present study, each long and narrow rice leaf was cut once transversely, the area of wounding was very small. No loss of turgidity in detached rice leaves treated with vanadate was observed. Therefore, the increase in ethylene production by vanadate could not result from water



Fig. 4. Effect of vanadate on the conversion of ACC to ethylene. Segments of rice leaves were pretreated with a saturating concentration of ACC $(10 \mathrm{mM})$ for $2 \mathrm{~h}$ and then treated with either water or $1 \mathrm{~m} M$ vanadate in darkness. Rates of ethylene production were determined at the times indicated. Bars represented standard errors $(n=4)$.

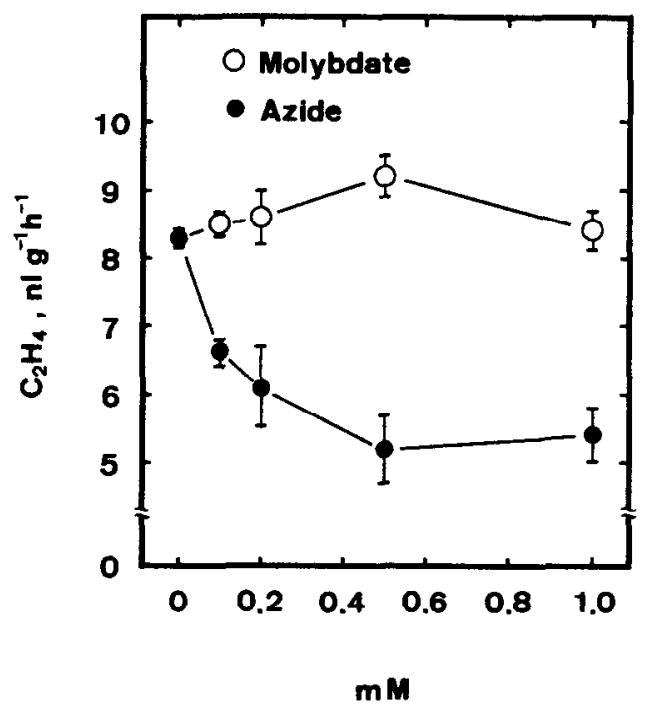

Fig. 5. Effect of molybdate and azide concentrations on ethylene production in segments of rice leaves. Ethylene production was assayed after $6 \mathrm{~h}$ incubation in darkness. Bars represented standard errors $(n=4)$.

stress. The effects of vanadate on the production of ethylene seem to be complex. At present, it is impossible to propose a general explanation for the stimulation by vanadate of the production of ethylene in rice leaf tissues and inhibition in other tissues. The discrepancy 


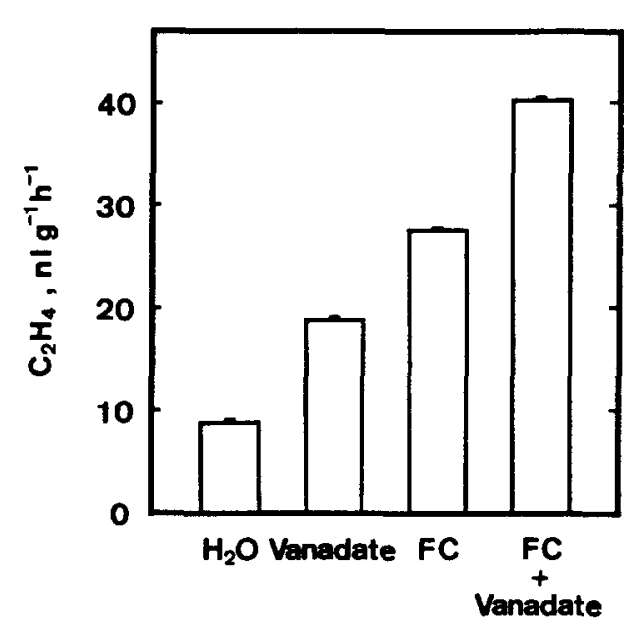

Fig. 6. Effect of FC on ethylene production and of FC on vanadate-induced ethylene production. Detached rice leaves were incubated in the dark for $6 \mathrm{~h}$ in vanadate $(1 \mathrm{mM}), \mathrm{FC}(1 \mu M)+$ vanadate $(1 \mathrm{mM})$. Bars represented standard errors $(n=4)$.

might be because different species respond to identical vanadate treatment in different ways.

Results from the present study clearly showed that vanadate induced a significant increase in the production of ethylene shortly (within $3 \mathrm{~h}$ ) after application. This observation suggests that vanadate stimulates biosynthesis of ethylene directly. Since senescence of rice leaves in darkness occurred at 2 days after incubation [2]. The vanadate-enhanced production of ethylene in detached rice leaves is unlikely to be mediated via mechanism associated with senescence.

Why is the extent of the stimulatory effect of vanadate on the production ethylene quite small, usually two-fold? One possibility is that vanadate is not readily transported in rice leaf cells. It should be noted that, in the present study, segments of unpeeled leaves were used.

The present investigation demonstrated that the increased production of ethylene in vanadate-treated leaves is regulated via promotion of the conversion of ACC to ethylene. However, it is unclear whether the increase in the activity of ACC oxidase is regulated by the de novo synthesis of ACC oxidase and/or by the activation of preexisting ACC oxidase.

FC has been shown to enhance ethylene production in detached rice leaves [3]. The present investigation showed that $F C$ and vanadate synergisticly increased the production of ethylene. Since FC and vanadate have opposite effects on $\mathrm{H}^{+}$-ATPase activity, it is unlikely that $\mathrm{H}^{+}$-ATPase is involved in the biosynthesis of ethylene in detached rice leaves.

\section{Acknowledgement}

The assistance of Miss YF Huang is gratefully acknowledged.

\section{References}

1. Bowman BJ, Mainzer SE, Allen $\mathrm{KE}$ and Slayman $\mathrm{CW}$ (1978) Effects of inhibitors on the plasma membrane and mitochondrial adenosine triphosphatases of Neurospora crassa. Biochim Biophys Acta 512: 13-28

2. Chen CT and Kao CH (1991) Senescence of rice leaves XXX. Levels of endogenous polyamines and dark induced senescence of rice leaves. Plant Cell Physiol 32: 935-941

3. Chen CT and Kao CH (1993) Characteristics of fusicoccioninduced production of ethylene in detached rice leaves. Plant Physiol Biochem 31: 121-124

4. Chen CT, Chou IT and Kao CH (1990) Senescence of rice leaves XX. Changes of poton secretion during senescence. Plant Sci 66: 29-34

5. Gallagher SR and Leonard RT (1982) Effect of vanadate, molybdate and azide on membrane-associated ATPase and soluble phosphatase activities of corn roots. Plant Physiol 70: 1335-1340

6. Huang $\mathrm{Y}$ and $\mathrm{Kao} \mathrm{CH}$ (1991) Senescence of rice leaves XXV III. Effect of molybdate and vanadate on acid phosphatase and leaf senescence. Bot Bull Acade Sin 32: 97-101

7. John P, Parter AJR and Miller AJ (1985) Activity of ethyleneforming enzyme measured in vivo at different cell potential. J Plant Physiol 121: 397-406

8. Kao $\mathrm{CH}$ and Yang SF (1982) Light inhibition of the conversion of 1 -aminocyclopropane-1-carboxylic acid to ethylene in leaves is mediated through carbon dioxide. Planta 155: 261266

9. Kelly MO and Bradford KJ (1990) Ethylene synthesis and growth of tomato hypocotyls: Induction of auxin and fusicoccin and inhibition by vanadate. Plant Growth Regul 9: 43-49

10. Marre E (1979) Fusicoccin: A tool in plant physiology. Annu Rev Plant Physiol 30: 273-288

11. Mayne RG and Kende $H$ (1986) Ethylene biosynthesis in isolated vacuoles of Vicia faba L. Requirement for membrane integrity. Planta 167: 159-165

12. O'Neill S and Spanswick RM (1984) Effect of vanadate on the plasma membrane ATPase of red beet and com. Plant Physiol 75: 586-591

13. Steffens M, Ettl F, Kranz D and Kindl H (1989) Vanadate mimics effects of fungal cell wall in eliciting gene entivation in plant cell cultures. Planta 177: 160-168

14. Yang SF and Hoffman NE (1984) Ethylene biosynthesis and its regulation in higher plants. Annu Rev Plant Physiol 35: 155-189

15. Zoglauer K, Dembny $\mathrm{H}$ and Goring $\mathrm{H}$ (1987) Inhibition of IAA-induced ethylene production and proline accumulation in wheat coleoptiles by fusicoccin. Biochem Physiol Pflanzen 182: $23-29$ 